\title{
REMARKS ON SUBHARMONIC ENVELOPES
}

\author{
DuONG NGOC SON
}

Abstract

We prove that the subharmonic envelope of a lower semicontinuous function on $\Omega$ is harmonic on a certain open subset of $\Omega$, using a very classical method in potential theory. The result gives simple proofs of theorems on harmonic measures and Jensen measures obtained by Cole and Ransford.

\section{Introduction and statement of results}

One of the most important objects of Classical Potential Theory is the Laplace equation

$$
\Delta h=\frac{\partial^{2} h}{\partial x_{1}^{2}}+\frac{\partial^{2} h}{\partial x_{2}^{2}}+\cdots+\frac{\partial^{2} h}{\partial x_{N}^{2}}=0
$$

in Euclidean space $\mathbb{R}^{N}$. Let $\Omega$ be a open subset of $\mathbb{R}^{N}$. A twice differentiable function $h$ defined on $\Omega$ is called harmonic if it satisfies the Laplace equation. The family of harmonic functions on $\Omega$ will be denoted by $\mathcal{H}(\Omega)$. The Dirichlet problem for the Laplace operator is the following: Given a continuous real-valued function $f$ on $\partial^{\infty} \Omega$ (i.e. the boundary of $\Omega$ in the one-point compactification of $\mathbb{R}^{N}$ ), find a harmonic function $h$ on $\Omega$ such that

$$
\lim _{y \rightarrow z} h(y)=f(z)
$$

for every $z \in \partial^{\infty} \Omega$.

The classical method of Perron-Wiener-Brelot for solving the Dirichlet problem is based on subharmonic functions. Recall that a function $u$ defined on $\Omega \subset \mathbb{R}^{N}$ with values in $[-\infty, \infty)$ is said to be subharmonic on $\Omega$ if $u$ is upper semicontinuous and for each $r>0$ small enough we

2000 Mathematics Subject Classification. 31B5.

Key words. Subharmonic envelope, harmonic measure, Jensen measure. 
have

$$
u(x) \leq \frac{1}{\lambda(B(x, r))} \int_{B(x, r)} u(t) d \lambda(t), \quad x \in \Omega .
$$

Here $d \lambda$ denotes Lebesgue measure in $\mathbb{R}^{N}$. Note that if $u$ is a harmonic function on $\Omega$, then equality holds in (1). Hence, any harmonic function is subharmonic. By $\mathcal{S H}(\Omega)$ we denote the family of subharmonic function on $\Omega$. For each bounded function $f$ defined on $\partial^{\infty} \Omega$ let

$$
\mathcal{U}(f ; \Omega)=\left\{v \in \mathcal{S H}(\Omega): \limsup _{y \rightarrow z} v(y) \leq f(z) \text { for each } z \in \partial^{\infty} \Omega\right\} .
$$

The Perron envelope of $f$ on $\Omega$ is

$$
H_{f}^{\Omega}(x):=\sup \{v(x): v \in \mathcal{U}(f ; \Omega)\} .
$$

It is well known that $H_{f}^{\Omega}$ is harmonic on $\Omega$. Moreover, if the Dirichlet problem has a solution, the solution must be $H_{f}^{\Omega}$. It is natural to ask under what conditions, $H_{f}^{\Omega}$ becomes the solution of the Dirichlet problem, i.e.

$$
\lim _{x \rightarrow z} H_{f}^{\Omega}(x)=f(z), \quad \forall z \in \partial \Omega .
$$

This problem leads us to the following notion. A point $z \in \partial^{\infty} \Omega$ is called a regular boundary point of $\Omega$ if (2) holds for all $f \in C\left(\partial^{\infty} \Omega\right)$. The classical theorem of O. D. Kellogg states that the set of irregular boundary points of $\Omega$ is a polar set (cf. [5, Theorem 8.34]).

Let $\Omega$ be an open subset of $\mathbb{R}^{N}$ and $x \in \Omega$. The mapping $L: C\left(\partial^{\infty} \Omega\right) \rightarrow$ $\mathbb{R}, f \mapsto H_{f}^{\Omega}(x)$ is a positive linear functional on the Banach space $C\left(\partial^{\infty} \Omega\right)$. By the well-known Riesz representation theorem there exists a unique positive Borel measure $\omega$ on $\partial^{\infty} \Omega$ such that for all $f \in C\left(\partial^{\infty} \Omega\right)$ we have

$$
\int_{\partial \infty \Omega} f d \omega=H_{f}^{\Omega}(x) .
$$

The measure $\omega$ above is called harmonic measure for $\Omega$ at $x$. By $H_{x}(\Omega)$ we denote the set of harmonic measures at $x$ with respect to relatively compact subdomains $\Omega^{\prime}$ of $\Omega$ (i.e. $\omega \in H_{x}(\Omega)$ if and only if there exists a relatively compact subdomain $\Omega^{\prime} \subset \Omega$ such that $\omega$ is the harmonic measure for $\Omega^{\prime}$ at $\left.x\right)$.

Let $\varphi$ be an arbitrary function on $\Omega$. The subharmonic envelope of $\varphi$

$$
S \varphi(x)=\sup \{v(x): v \text { is subharmonic and } v \leq \varphi \text { on } \Omega\} .
$$

Subharmonic envelopes have been studied extensively. They became an important tool in classical potential theory. It is well known that 
the upper semicontinuous regularization $(S \varphi)^{*}$ of $S \varphi$ is a subharmonic function, provided that it is locally bounded above. Moreover, we have the following

Cartan's Theorem. Let $U \subset \mathcal{S H}(\Omega)$ and let $u:=\sup _{v \in U} v$. Assume that $u$ is locally bounded above. Then $u^{*} \in \mathcal{S H}(\Omega)$ and $u^{*}=u$ outside a polar set.

Recall that a subset $E$ of $\Omega$ is said to be polar if there exists a subharmonic function $v$ on $\Omega, v \not \equiv-\infty$ such that $E \subset\{x \in \Omega: v(x)=-\infty\}$. If $w$ is locally bounded above then the upper semicontinuous regularization $w^{*}$ of $w$ is defined as follows.

$$
w^{*}(x)=\limsup _{y \rightarrow x, y \in \Omega} w(y), \quad x \in \Omega .
$$

It is easy to see that $w^{*}$ is upper semicontinuous and $w^{*} \geq w$. Moreover, $w^{*}$ is the smallest function with these properties.

Recently, Cole and Ransford studied quasi-subharmonic functions on an open subset of $\mathbb{R}^{N}$. It turns out from their results that the class of subharmonic envelopes is nothing but the class of quasi-subharmonic functions [2, Theorem 1.1, Theorem 1.5]. They also proved the following [2, Corollary 1.7].

Theorem 1. Let $\Omega$ be an open subset of $\mathbb{R}^{N}$ which possesses a Green function. Let $\varphi: \Omega \rightarrow[-\infty, \infty)$ be a measurable function which is locally bounded above. Then for each $x \in \Omega$

$$
S \varphi(x)=\inf \left\{\int \varphi d \mu: \mu \text { is a Jensen measure for } x\right\} \text {. }
$$

Recall that a Jensen measure for $x$ is a Borel measure $\mu$, supported on a compact subset of $\Omega$ such that each subharmonic function $u$ on $\Omega$ satisfies

$$
u(x) \leq \int u d \mu
$$

By $J_{x}(\Omega)$ we mean the set of all Jensen measures at $x$ with respect to $\Omega$. The family of Jensen measures for $x$ contains the subfamily $H_{x}(\Omega)$ of harmonic measures at $x$ with respect to some relatively compact subdomain $D$ of $\Omega$ (cf. [3, Proposition 3.1]). Moreover, we have the following relation between the two families (cf. [3, Theorem 1.3]). 
Theorem 2. Let $\Omega$ be an open subset of $\mathbb{R}^{N}$. Let $\varphi: \Omega \rightarrow[-\infty, \infty)$ be a measurable function which is locally bounded above. Then for each $x \in \Omega$

(6) $\inf \left\{\int \varphi d \mu: \mu \in J_{x}(\Omega)\right\}=\inf \left\{\int \varphi d \omega: \omega \in H_{x}(\Omega) \cup\left\{\delta_{x}\right\}\right\}$.

One aim of the paper is to prove some results concerning the two classes of measures. We rely on the following main result which seems to be classical, but we cannot find it in the literature.

Theorem 3. Let $\Omega$ be a bounded open subset of $\mathbb{R}^{N}$ and $\varphi: \bar{\Omega} \rightarrow \mathbb{R}$ a bounded, lower semicontinuous function. Denote by $S \varphi$ its subharmonic envelope. Let $W=\left\{y \in \Omega:(S \varphi)^{*}(y)<\varphi(y)\right\}$. Then $(S \varphi)^{*}$ is subharmonic on $\Omega$, harmonic on $W$ and for each regular boundary point $z \in \partial \Omega$ the following inequality holds.

$$
\liminf _{y \rightarrow z}(S \varphi)^{*}(y) \geq \varphi(z) .
$$

This result gives simple proofs and sometimes stronger statements of some theorems on harmonic measures and Jensen measures obtained in $[\mathbf{3}]$.

\section{Proof and applications}

We need the following

Lemma 4. Let $\Omega$ be an open subset of $\mathbb{R}^{N}$ which possesses a Green function and $E \subset \Omega$ a polar set. Given $x_{0} \in \Omega$, there exists a subharmonic function $v$ on $\Omega$ such that $v<0$ on $\Omega, v \equiv-\infty$ on $E \backslash\left\{x_{0}\right\}$, $v\left(x_{0}\right)=-1$.

Proof: Cf. [1, Theorem 5.1.3].

Proof of Theorem 3: First, we prove (7). Let $z \in \partial \Omega$ be a regular boundary point of $\Omega$. Given $\epsilon>0$, since $\varphi$ is lower semicontinuous, there exists $r_{0}>0$ such that $\varphi(y)>\varphi(z)-\epsilon$ for all $y \in B\left(z, r_{0}\right) \cap \bar{\Omega}$. Consider the function $m$ on $\partial \Omega$ defined by

$$
m(y)=r_{0}^{-1}\|y-z\| .
$$

Since $z$ is regular, we have

$$
\lim _{x \rightarrow z} H_{m}^{\Omega}(x)=m(z)=0 .
$$

Note that the function $v$ on $\Omega, v(y)=r_{0}^{-1}\|y-z\|$ is subharmonic and $\lim _{y \rightarrow x} v(y)=m(x)$ for all $x \in \partial \Omega$, so $v \in \mathcal{U}(m ; \Omega)$. Therefore $v \leq H_{m}^{\Omega}$ on $\Omega$. 
Now consider the function

$$
\psi(y)=-H_{m}^{\Omega}(y)(\varphi(z)-M+1)+\varphi(z)-\epsilon,
$$

where $M=\inf \{\varphi(x): x \in \Omega\}$. For each $y \in \Omega \backslash B\left(z, r_{0}\right)$ we have

$$
H_{m}^{\Omega}(y) \geq v(y)=r_{0}^{-1}\|y-z\| \geq 1
$$

and therefore

$$
\begin{aligned}
\psi(y) & \leq-(\varphi(z)-M+1)+\varphi(z)-\epsilon \\
& =M-1-\epsilon \\
& <\varphi(y) .
\end{aligned}
$$

Otherwise, if $y \in B\left(z, r_{0}\right) \cap \Omega$, then $\psi(y) \leq \varphi(z)-\epsilon<\varphi(y)$ since $H_{m}^{\Omega}>0$. Hence, $\psi<\varphi$ on $\Omega$. Moreover, since $\psi \in \mathcal{S H}(\Omega)$, we have $\psi \leq S \varphi$ on $\Omega$ and therefore, by (8),

$$
\begin{aligned}
\liminf _{y \rightarrow z}(S \varphi)^{*}(y) & \geq \liminf _{y \rightarrow z} \psi(y) \\
& \geq\left[\liminf _{y \rightarrow z}\left(-H_{m}^{\Omega}(y)\right)\right](\varphi(z)-M+1)+\varphi(z)-\epsilon \\
& =\varphi(z)-\epsilon .
\end{aligned}
$$

As $\epsilon>0$ is arbitrary, we have $\liminf _{y \rightarrow z}(S \varphi)^{*}(y) \geq \varphi(z)$, as desired.

By Cartan's Theorem, $(S \varphi)^{*}$ is a subharmonic function on $\Omega$ and $(S \varphi)^{*}=S \varphi$ outside a polar set. It remains to show that $(S \varphi)^{*}$ is a harmonic function on $W$. Let $x \in W$, it suffices to show that there exists $r>0$ such that $(S \varphi)^{*}$ is harmonic on the ball $B(x, r)$.

Since $x \in W$ then $(S \varphi)^{*}(x)=\varphi(x)-2 \delta$ for some $\delta>0$. On the other hand, $(S \varphi)^{*}$ is upper semicontinuous and $\varphi$ is lower semicontinuous, so we can find $r>0$ such that for all $y \in \bar{B}=\overline{B(x, r)}$ we have

$$
(S \varphi)^{*}(y)<\varphi(x)-\delta, \quad \varphi(y)>\varphi(x)-\delta .
$$

By the Poisson Modification theorem, there exists $s \in \mathcal{S H}(\Omega)$ such that $s \geq(S \varphi)^{*}, s=(S \varphi)^{*}$ on $\Omega \backslash B$ and $s$ is harmonic in $B$. To complete the proof, we need only to show that $s \leq(S \varphi)^{*}$ on $B$. Indeed, let $x_{0} \in B$ and $\epsilon>0$ be arbitrary. First, $s \leq \varphi$ on $B$ since by the maximum principle,

$$
s(y) \leq \sup _{z \in \partial B}(S \varphi)^{*}(z) \leq \varphi(x)-\delta<\varphi(y), \quad(\forall y \in B) .
$$

Take $E=\left\{y \in \Omega:(S \varphi)^{*}(y)>\varphi(y)\right\}$. Then

$$
E \subset\left\{y \in \Omega:(S \varphi)^{*}(y) \neq S \varphi(y)\right\} .
$$


By Cartan's Theorem, the set

$$
\left\{y \in \Omega:(S \varphi)^{*}(y) \neq S \varphi(y)\right\}
$$

is a polar set and therefore $E$ is polar. By Lemma 4, there exists $u \in$ $\mathcal{S H}(\Omega)$ such that $u \leq 0$ on $\Omega, u\left(x_{0}\right)=-1$ and $\left.u\right|_{E} \equiv-\infty$. Put $w=s+\epsilon u$. Then $w \in \mathcal{S H}(\Omega)$. We will show that $w<\varphi$ on $\Omega$ so that $w \leq S \varphi$ on $\Omega$ and in particular,

$$
s\left(x_{0}\right)-\epsilon=s\left(x_{0}\right)+\epsilon u\left(x_{0}\right)=w\left(x_{0}\right) \leq S \varphi\left(x_{0}\right) \leq(S \varphi)^{*}\left(x_{0}\right) .
$$

Now it remains to check that $w<\varphi$ on $\Omega$. Let $y \in \Omega$. Consider three cases:

1. If $y \in B$, then $w(y)<s(y)<\varphi(y)$, since $u(y)<0$ and (9) holds.

2. If $y \in E$, then $w(y)=-\infty$, since $\left.u\right|_{E} \equiv-\infty$.

3. If $y \in \Omega \backslash(B \cup E)$, then by definition of $E$ we have

$$
w(y)<s(y)=(S \varphi)^{*}(y) \leq \varphi .
$$

Therefore $w<\varphi$ on $\Omega$. Hence $s\left(x_{0}\right)-\epsilon \leq(S \varphi)^{*}\left(x_{0}\right)$, where $\epsilon>0$ and $x_{0} \in B$ are arbitrary. Thus $s=(S \varphi)^{*}$ on $B$ and therefore $(S \varphi)^{*}$ is harmonic on $B$, so the proof is completed.

Remark. If $W$ is empty, then the theorem is rather trivial. Otherwise, $W$ is an open subset of $\Omega$ so the harmonicity of $(S \varphi)^{*}$ on $W$ makes sense; also $S \varphi=(S \varphi)^{*}$ on $W$.

Remark. It is natural to ask if Theorem 3 is true without the semicontinuity of $\varphi$. In this case, since $W$ is not an open set, one cannot discuss harmonicity of $(S \varphi)^{*}$. But we can rephrase the harmonicity in term of Riesz measure. Thus we can expect that the Riesz measure associated with $(S \varphi)^{*}$ puts zero mass on $W$ (or on a certain subset of $W$ ). More precisely, we formulate the following question.

Question. We keep the assumptions in Theorem 3 except that the semicontinuity of $\varphi$ is replaced by Borel measurability. Does $\mu_{(S \varphi)^{*}}(U)=0$, where $\mu_{(S \varphi)^{*}}$ is the Riesz measure associated with $(S \varphi)^{*}$ and $U$ is the fine interior of $W$ ?

It would need more analysis in fine topology (see [1, Chapter 7$]$ for basic concepts of fine topology) to prove such a result. But Theorem 3 is enough for our applications.

We have the following corollary which should be compared with Lemma 3.3 in $[\mathbf{3}]$. 
Corollary 5. Let $\Omega$ be an open subset of $\mathbb{R}^{N}$ which possesses a Green function and $x \in \Omega$. Let $\varphi$ be a continuous function on $\Omega \cup \partial^{\infty} \Omega$ and $S \varphi$ be its subharmonic envelope. Assume that $S \varphi(x)<\varphi(x)$. Then there exists a domain $D \subset \Omega$ such that $x \in D$ and

$$
\int \varphi d \omega \leq S \varphi(x)
$$

where $\omega$ is the harmonic measure for $D$ at $x$.

Proof: First we assume that $\Omega$ is bounded. Let $W=\{y \in \Omega: S \varphi(y)<$ $\varphi(y)\}$. Since $S \varphi(x)<\varphi(x)$ then $x \in W$. Let $D$ be the connected component of $W$ containing $x$ and $\omega$ the harmonic measure for $D$ at $x$. By Theorem 3, $(S \varphi)^{*}=S \varphi$ and it is harmonic on $D$. Moreover, for any regular boundary point $z$ of $\Omega$ such that $z \in \partial D$, we have

$$
\liminf _{y \rightarrow z, y \in D} S \varphi(y) \geq \varphi(z) .
$$

On the other hand, since $\varphi$ is continuous,

$$
\limsup _{y \rightarrow z} S \varphi(y) \leq \limsup _{y \rightarrow z} \varphi(y)=\varphi(z) .
$$

Hence

$$
\lim _{y \rightarrow z, y \in D} S \varphi(y)=\varphi(z) .
$$

For $z \in \partial D \cap \Omega$ which is regular with respect to $D$, we can use an argument similar to the proof of Theorem 3 to show that

$$
\liminf _{y \rightarrow z} S \varphi(y)=\varphi(z) \text {. }
$$

By Kellogg's Theorem (cf. [4, Theorem 5.20]), the set of irregular boundary points is polar. Hence (12) holds for $z \in \partial D$ outside a polar set. Therefore, by the generalized maximum principle, we have (10).

Now for general case we need the following classical lemma.

Lemma 6. Let $\left\{D_{n}\right\}$ be an increasing sequence of subdomains of a domain $D$ such that $\bigcup_{n=1}^{\infty} D_{n}=D$ and $x \in D_{1}$. If $f$ is a continuous function on $D \cup \partial^{\infty} D$ and $\omega_{n}, \omega$ are harmonic measures at $x$ for $D_{n}, D$ respectively, then

$$
\int_{\partial^{\infty} D} f d \omega_{n} \longrightarrow \int_{\partial^{\infty} D} f d \omega \text { as } n \rightarrow \infty .
$$

Proof: It follows from Theorem 6.3.10 in [1] that $H_{f}^{D_{n}}(x) \rightarrow H_{f}^{D}(x)$ as $n \rightarrow \infty$. Then the proof is completed since

$$
H_{f}^{D_{n}}(x)=\int_{\partial^{\infty} D} f d \omega_{n} \text { and } H_{f}^{D}(x)=\int_{\partial^{\infty} D} f d \omega .
$$


For $j>0$ let $\Omega_{j}=\Omega \cap B(0, j)$. We can assume that $\Omega_{j}$ is non-empty for all $j$. Clearly, $\bigcup_{j} \Omega_{j}=\Omega$. Let

$$
w_{j}(x)=\sup \left\{v(x): v \text { is subharmonic on } \Omega_{j} \text { and } v \leq \varphi \text { on } \Omega_{j}\right\}
$$

and $W_{j}=\left\{y \in \Omega_{j}: w_{j}(y)<\varphi(y)\right\}$. It is easy to see that the sequence $\left\{w_{j}\right\}$ decreases to $S \varphi$. Therefore $W=\bigcup_{j} W_{j}$. Also, $x \in W_{j}$ for $j$ large enough so we can assume that $x \in W_{j}$ for all $j$. Let $D_{j}$ be the connected component of $W_{j}$ containing $x$. Clearly $D=\bigcup_{j} D_{j}$ is the connected component of $W$ containing $x$. By what we have already proved, we have

$$
\int \varphi d \omega_{j} \leq w_{j}(x)
$$

where $\omega_{j}$ is the harmonic measure for $D_{j}$ at $x$. Let $j \rightarrow \infty$. Then (10) follows from Lemma 6 . The proof is completed.

By $H_{x}^{s}(\Omega)$ we denote the subclass of $H_{x}(\Omega)$ consisting of all harmonic measures of domains $D \Subset \Omega$ with $C^{\infty}$-smooth boundary. We prove the following improvement of Theorem 1.3 in $[\mathbf{3}]$.

Theorem 7. Let $\Omega$ be an open subset of $\mathbb{R}^{N}$ which possesses a Green function and $x \in \Omega$. Suppose that $\varphi: \Omega \rightarrow \mathbb{R}$ be an upper semicontinuous function. Then

$$
S \varphi(x)=\inf \left\{\int \varphi d \omega: \omega \in H_{x}^{s}(\Omega)\right\} .
$$

Remark. It follows from [3, Proposition 4.1] that there exists a lower semicontinuous function $\varphi$ such that (13) does not hold.

Proof: It follows from [3, Proposition 3.1] that $H_{x}^{s}(\Omega) \subset J_{x}(\Omega)$. Then

$$
S \varphi(x) \leq \inf \left\{\int \varphi d \omega: \omega \in H_{x}^{s}(\Omega)\right\} .
$$

To show that in fact equality holds, first assume that $\varphi$ is a continuous function on $\Omega \cup \partial^{\infty} \Omega$. It is enough to show that for all $x \in \Omega$ there exist subdomains $D_{n} \Subset \Omega$ with $C^{\infty}$-smooth boundaries such that

$$
S \varphi(x) \geq \lim _{j \rightarrow \infty} \int \varphi(\xi) d \omega\left(D_{j}, x, \xi\right) .
$$


Indeed, if $S \varphi(x)<\varphi(x)$ then, by Corollary 5, there exists subdomain $D \subset \Omega$ such that

$$
S \varphi(x) \geq \int \varphi(\xi) d \omega(D, x, \xi),
$$

where $\omega(D, x, \cdot)$ is the harmonic measure for $D$ at $x$. Take a sequence $\left\{D_{j}\right\}_{j=1}^{\infty}$ of $C^{\infty}$-smooth domains such that $D_{j} \Subset D$ and $D_{j} \nearrow D$. Then

$$
S \varphi(x) \geq \int \varphi(\xi) d \omega(D, x, \xi)=\lim _{j \rightarrow \infty} \int \varphi(\xi) d \omega\left(D_{j}, x, \xi\right) .
$$

Then (14) holds. Otherwise, we can take $\left\{D_{j}\right\}_{j=1}^{\infty}$ to be a sequence of small balls centered at $x$ with radii decreasing to 0 . Clearly

$$
S \varphi(x) \geq \varphi(x)=\lim _{j \rightarrow \infty} \int \varphi(\xi) d \omega\left(D_{j}, x, \xi\right) .
$$

Hence the theorem is true in this case.

Now we turn to general case. Let $\left\{\varphi_{j}\right\}_{j=1}^{\infty}$ be a sequence of continuous functions on $\Omega \cup \partial^{\infty} \Omega$ decreasing to $\varphi$. Then $S \varphi_{j}$ decreases to a subharmonic function $v$. Clearly $v \leq \varphi_{j}$ for all $j$ and therefore $v \leq \varphi$. Hence $v \leq S \varphi$. On the other hand, $S \varphi \leq S \varphi_{j}$ for all $j$. Therefore $S \varphi \leq v$ and hence $v=S \varphi$. Moreover

$$
\begin{aligned}
v(x)=\lim _{j \rightarrow \infty} S \varphi_{j}(x) & =\lim _{j \rightarrow \infty}\left(\inf \left\{\int \varphi_{j} d \omega: \omega \in H_{x}^{s}(\Omega)\right\}\right) \\
& \geq\left\{\int \varphi d \omega: \omega \in H_{x}^{s}(\Omega)\right\} .
\end{aligned}
$$

This completes the proof.

Remark. In this theorem, one can replace the family $H_{x}^{s}(\Omega)$ by any family of harmonic measures of domains which can approximate general domains; for example, one can use $H_{x}^{p}(\Omega)$ the family of harmonic measures at $x$ with respect to polygons instead of $H_{x}^{s}(\Omega)$.

The sets $J_{x}(\Omega)$ and $H_{x}^{s}(\Omega)$ can be considered as subsets of the dual space $C^{*}(\Omega)$ of the space of continuous functions on $\Omega$, equipped with the weak-star topology. It is shown by Cole and Ransford in $[\mathbf{3}]$ that $J_{x}(\Omega)$ is a closed convex set and

$$
J_{x}(\Omega)=\overline{\operatorname{conv}\left(H_{x}(\Omega)\right)} .
$$

By using their arguments, we have the following corollary.

Corollary 8. Let $\Omega \subset \mathbb{R}^{N}$ be an open set. Then $J_{x}(\Omega)=\overline{\operatorname{conv}\left(H_{x}^{s}(\Omega)\right)}$, where the right-hand side is the closed convex hull of $H_{x}^{s}(\Omega)$. 
Acknowledgements. These results have their origin in my graduation paper on Jensen measures and harmonic measures, written under the direction of Nguyen Quang Dieu at Ha Noi University of Education, Vietnam. The author would like to thank him for giving me this topic, great help and valuable comments. Also the author would like to thank the referee for her/his valuable comments made on the first version of this paper.

\section{References}

[1] D. H. Armitage and S. J. Gardiner, "Classical potential theory", Springer Monographs in Mathematics, Springer-Verlag London, Ltd., London, 2001.

[2] B. J. Cole and T. J. Ransford, Subharmonicity without upper semicontinuity, J. Funct. Anal. 147(2) (1997), 420-442.

[3] B. J. Cole And T. J. Ransford, Jensen measures and harmonic measures, J. Reine Angew. Math. 541 (2001), 29-53.

[4] W. K. Hayman and P. B. Kennedy, "Subharmonic functions", Vol. I, London Mathematical Society Monographs 9, Academic Press [Harcourt Brace Jovanovich, Publishers], London-New York, 1976.

[5] L. L. HeLms, "Introduction to potential theory", Reprint of the 1969 edition, Pure and Applied Mathematics XXII, Robert E. Krieger Publishing Co., Huntington, N.Y., 1975.

Department of Mathematics

Ha Noi University of Education

136 Xuan Thuy, Cau Giay, Ha Noi

Vietnam

E-mail address: duongngocson@gmail.com

Primera versió rebuda el 3 d'octubre de 2005, darrera versió rebuda el 13 de febrer de 2006. 